\title{
The Pore Architecture of the Cystic Fibrosis Transmembrane Conductance Regulator Channel Revealed by Co-Mutation in Pore-Forming Transmembrane Regions
}

\author{
Feng QIAN ${ }^{1,2}$, Lian LIU ${ }^{1,2}$, Zhenzhen LIU², Chengbiao LU' ${ }^{1}$ \\ ${ }^{1}$ Laboratory of Neuronal Network and Brain Diseases Modulation, Yangtze University, Jingzhou, \\ Hubei Province, China, ${ }^{2}$ Department of Medical Function, School of Medicine, Yangtze University, \\ Jingzhou, Hubei Province, China
}

Received July 16, 2015

Accepted January 8, 2016

On-line April 12, 2016

\section{Summary}

The cystic fibrosis transmembrane conductance regulator (CFTR) chloride channel contains 12 transmembrane (TM) regions that are presumed to form the channel pore. However, there is no direct evidence clearly illustrating the involvement of these transmembrane regions in the actual CFTR pore structure. To obtain insight into the architecture of the CFTR channel pore, we used patch clamp recording techniques and a strategy of comutagenesis of two potential pore-forming transmembrane regions (TM1 and TM6) to investigate the collaboration of these two TM regions. We performed a range of specific functional assays comparing the single channel conductance, anion binding, and anion selectivity properties of the co-mutated CFTR variants, and the results indicated that TM1 and TM6 play vital roles in forming the channel pore and, thus, determine the functional properties of the channel. Furthermore, we provided functional evidence that the amino acid threonine (T338) in TM6 has synergic effects with lysine (K95) in TM1. Therefore, we propose that these two residues have functional collaboration in the CFTR channel pore and may collectively form a selective filter.

\section{Key words}

Cystic fibrosis transmembrane conductance regulator (CFTR) • Patch clamp • Site-directed mutagenesis

\section{Corresponding author}

C. Lu, Laboratory of Neuronal Network and Brain Diseases Modulation, Yangtze University, 1 Nanhuan Road, Jingzhou, Hubei province, China, 434023. E-mail: johnlu9000@hotmail.com

\section{Introduction}

The individual cystic fibrosis transmembrane conductance regulator (CFTR) is a chloride channel that has two hydrophobic core regions, each with six membrane spanning regions and two nucleotide binding domains (NBDs) (Sheppard and Welsh 1999). A large cytoplasmic regulatory domain connects the two halves of the CFTR molecule. CFTR is activated by phosphorylation of the regulatory domain via cAMPactivated protein kinase A (PKA) and via ATP binding and hydrolysis at the NBDs (Gadsby et al. 1998, Vergani et al. 2005). Mutations in CFTR lead to defective regulation or inhibit the transport of chloride ions across the apical surface of epithelial cells and are the primary cause of the lethal genetic disease cystic fibrosis (Anderson et al. 1991, Pilewski and Frizzell 1999). Despite progress in elucidating the domains involved in the function of CFTR channels, our understanding of the structural basis for chloride conductance through CFTR channels is incomplete. Fundamental questions regarding the CFTR pore architecture, anion binding and selectivity filter remain unanswered.

Mutations in amino acid residues that are conserved in the CFTR generate significant alterations in channel activity and anion permeation; thus, these mutations are associated with cystic fibrosis (Dawson et al. 1999). As such, site-directed mutagenesis has been widely employed as a means of creating clinically important mutants and obtaining information about the 
functional significance of amino acid residues in the CFTR chloride channel. In cation channels, the cationselective pore contains re-entrant loops (Dutzler et al. 2002, 2003, MacKinnon 2003). However, the structurefunction studies of anion channel pores have mainly focused on the transmembrane regions, which are presumed to form the physical pores of the anion channels. A number of laboratories have used mutagenesis to identify amino acid residues that are critical for CFTR chloride channel conductance and anion permeation (Ge et al. 2004, El Hiani and Linsdell 2010, Bai et al. 2011, Qian et al. 2011). It is apparent that many pore-lining residues (R334, K335, F337, T338, S341, I344, V345, R347, and R352) have been identified in TM6, which is the most studied transmembrane region, and TM6 is the TM region that has most clearly been demonstrated to be important for CFTR pore properties (Dawson et al. 1999, McCarty and Zhang 2001, Bai et al. 2010, El Hiani and Linsdell 2010). In recent years, studies of a cysteine-less CFTR variant have generated evidence of the involvement of TM1 (Wang et al. 2011, Gao et al. 2013), TM11 (Wang et al. 2014) and TM12 (Bai et al. 2011, Qian et al. 2011, Cui et al. 2012) in the CFTR pore properties. Among these transmembrane regions, TM1 is the most likely to cooperate with TM6 in channel pore formation (Akabas et al. 1994, Ge et al. 2004, Linsdell 2006, Wang et al. 2011), and substitutions of several amino acid residues, such as G91 (Akabas et al. 1994, Mansoura et al. 1998), K95 (Anderson et al. 1991, Ge et al. 2004), A96 (Ge et al. 2004), Q98 (Akabas et al. 1994, Ge et al. 2004), and P99 (Sheppard et al. 1996, Ge et al. 2004), have been shown to affect the functional properties of the CFTR channel. However, the mutation-induced CFTR functional changes in the cysteine-less CFTR variant provide little direct insight into the structural features of this channel, such as the CFTR pore architecture (Linsdell 2014a).

Previous findings have demonstrated that TM1 and TM6 are major contributors to the CFTR channel pore and have strong implications for emerging structural models of CFTR proteins (Linsdell 2014b, Wang et al. 2014). To test the hypothesis that TM1 and TM6 are physically closely located within the CFTR protein and confirm that TM1 and TM6 are involved in CFTR pore formation, the current study applied a co-mutagenesis strategy based on the following intuitive principle. If the two mutated residues are functionally independent, the double mutant (one residue in TM1 and TM6 each) should demonstrate an additive effect of the two single mutants on the functional properties of the CFTR channel. In contrast, if the two residues are located close enough to have a strong and specific collaboration with each other, a synergic outcome is expected. For example, through co-mutagenesis, we previously found that simultaneous mutations of two close residues (T338A/T339A or T338A/S341A) near the middle of TM6 significantly changed single channel conductance: the conductance was greater for the T338A/T339A double mutant than for the associated single mutants (T338A and T339A), and the conductance was less for the T338/S341A double mutant than for the associated single mutants (T338A and S341A) (Qian et al. 2014). Another study showed dynamic interaction between two CFTR residues (R555 and T1246) that were expected to lie on opposite sides of the predicted NBD1-NBD2 dimer interface (Vergani et al. 2005). This unexpected result caused by simultaneous mutations of two neighbor residues is a good example of residue collaboration when residues are located close to each other. Based on this assumption, in the present study, we investigated whether a synergic effect exists between two CFTR residues, one in TM1 and one in TM6. The T338A-CFTR channel (Linsdell et al. 1998, Ge et al. 2004, Fatehi et al. 2007) has outstanding functional properties because mutation of threonine to alanine causes T338A-CFTR channels to have highly characteristic functional properties of CFTR channels in terms of single channel conductance (Fatehi et al. 2007, Qian et al. 2014), anion permeability (Fatehi et al. 2007) and channel blocker binding affinity (Gong et al. 2002b, Fatehi et al. 2007); therefore, the T338A mutant was selected as the background variant for the characterization of the effects of the double mutants (with an additional mutation in TM1). The site in TM1 that was most highly expected to collaborate with T338 was lysine at position 95 (K95) because K95 is located at the same layer as T338 when TM1 and TM6 are arranged antiparallel (Ge et al. 2004). Here, we chose three residues in TM1 for mutagenesis, K95, A96 and Q98, as they had previous been proposed to be pore-lining residues (Ge et al. 2004, Linsdell 2014a). Finally, three double mutants (K95Q/T338A, A96V/T338A, and Q98A/T338A) were constructed, and their functional properties were compared to those of wild type CFTR and four single CFTR mutants (T338A, K95Q, A96V, and Q98A). 


\section{Methods}

The pIRES2-EGFP-CFTR plasmid was kindly provided by Dr. Paul Linsdell (Dalhousie University, Halifax, Canada). Site-directed mutagenesis of CFTR was conducted using the QuikChange kit (Agilent Technologies, La Jolla, CA, USA). Following long-chain PCR, the DNA product was treated with $D p n \mathrm{I}$ for $2 \mathrm{~h}$ at $37^{\circ} \mathrm{C}$ and was then transformed into competent Escherichia coli cells and grown overnight on LB agar plates containing $30 \mu \mathrm{g} \mathrm{ml}^{-1}$ kanamycin (Life Technologies, Carlsbad, CA, USA). Six separate colonies were selected and expanded, and the plasmids were purified for confirmation of the presence of the desired mutation by DNA sequencing. Experiments were carried out on Chinese hamster ovary $(\mathrm{CHO})$ and baby hamster kidney (BHK) cells (ATCC, Manassas, VA, USA) transiently transfected with wild type or mutated CFTR plasmids. For the patch clamp recordings, the cells were seeded onto $22 \mathrm{~mm}$ glass cover slips in $35 \mathrm{~mm}$ culture dishes, grown overnight and transfected using a Lipofectamine kit (Life Technologies, Carlsbad, CA, USA). Transiently transfected $\mathrm{CHO}$ and BHK cells were identified by fluorescence microscopy within $24 \mathrm{~h}$ and were used for patch clamp recording within 3 days after transfection.

Electrophysiological data were recorded from excised, inside-out patches of the cell membranes of the transfected $\mathrm{CHO}$ and BHK cells. Briefly, CFTR channels were activated after $\mathrm{CHO}$ cell patch excision by exposure to $5 \mathrm{nM}$ of the catalytic subunit of protein kinase A (PKA) and $2 \mathrm{mM} \mathrm{MgATP}$ for unitary trace recordings. The macroscopic currents were recorded in inside-out patches of transfected BHK cells following maximal channel activation in the presence of $2 \mathrm{mM}$ ATP, $50 \mathrm{nM}$ PKA and $2 \mathrm{mM}$ pyrophosphate, a nucleotide analogue, to keep the channels in the open configuration (Gunderson and Kopito 1994). For single channel recording, the extracellular (pipette) solution contained $150 \mathrm{mM}$ sodium gluconate, $10 \mathrm{mM} \quad \mathrm{N}$-tris[hydroxymethyl] methyl-2aminoethanesulfonate (TES) and $2 \mathrm{mM} \mathrm{MgCl}_{2}$, and the intracellular solution contained $150 \mathrm{mM} \mathrm{NaCl}, 10 \mathrm{mM}$ TES and $2 \mathrm{mM} \mathrm{MgCl}_{2}$. To estimate anion permeability, $\mathrm{NaCl}$ in the extracellular solution was replaced with $150 \mathrm{mM} \mathrm{NaSCN}$ or $150 \mathrm{mM} \mathrm{NaBr}$. For the iodide permeability experiment, $150 \mathrm{mM} \mathrm{NaCl}$ in the intracellular solution was replaced with $150 \mathrm{mM} \mathrm{NaI}$ to avoid electrode oxidation in the pipette. All of the solutions were adjusted to a $\mathrm{pH}$ of 7.4 with $\mathrm{NaOH}$. The given voltages were corrected for liquid junction potentials using Clampex10.3 software (Molecular Devices, Sunnyvale, CA, USA). The pipette resistances were 6-10 M $\Omega$ for the single channel recordings and approximately $2 \mathrm{M} \Omega$ for the macroscopic excised patch recording. Currents were filtered at $50 \mathrm{~Hz}$ for single channel recordings and $1 \mathrm{kHz}$ for macroscopic recordings using an 8-pole Bessel filter (Frequency Devices, Ottawa, Canada), were amplified using an Axopatch 700B amplifier (Molecular Devices, Sunnyvale, CA, USA), were digitized at $250 \mathrm{~Hz}$ for single channel recordings and $5 \mathrm{kHz}$ for macroscopic recordings using a DigiData 1440A digitizer and were analyzed using Clampfit10.3 software (Molecular Devices, Sunnyvale, CA, USA). Unless stated otherwise, all chemicals were purchased from Sigma-Aldrich, USA.

Macroscopic current-voltage $(I-V)$ relationships were constructed using depolarizing voltage ramp protocols as described previously (Gong and Linsdell 2003a, Ge et al. 2004). Macroscopic currents from $-100 \mathrm{mV}$ to $60 \mathrm{mV}$ were elicited within $500 \mathrm{~ms}$ using the voltage ramp protocol. Current amplitude was measured at the beginning $(20 \mathrm{~ms})$ and end $(480 \mathrm{~ms})$ of the voltage ramp protocol. The $20 \mathrm{~ms}$-time point was selected to ensure that the cell membrane capacitive current did not contribute to the measured current amplitude and was used to calculate the inhibition ratio of $\mathrm{Au}(\mathrm{CN})_{2}{ }^{-}$ blockade and the rectification ratio. Currents were measured in the absence of PKA and ATP and were subtracted from the currents measured in the presence of PKA and ATP. The experiments were performed at room temperature. Values are presented as means \pm SEM. Statistical comparisons between wild type CFTR and mutants were carried out using Student's two-tailed $t$-test. The differences among single and double mutated CFTR channels were analyzed using one-way ANOVA and post-hoc test (Turkey HSD). $P<0.05$ was considered to indicate a statistically significant difference.

\section{Results}

Single channel properties of single and double mutants

Expression of wild type or mutant CFTRs in Chinese hamster ovary (CHO) cells led to the appearance of PKA- and ATP-dependent chloride channel currents in excised inside-out membrane patches (Fig. 1). For the single mutants, A96V and Q98A showed similar unitary currents to that of the wild type CFTR, as indicated by the all-points histograms (right panel in Fig. 1). However, the unitary conductance was drastically reduced in the K95Q 
mutant (Fig. 1). In contrast, T338A led to a significant increase in channel conductance, as described previously (Linsdell et al. 1998, Qian et al. 2014). For the double mutant (A96V/T338A), the enhancive effect of the T338A single mutation on the unitary current was mitigated by the co-mutation of A96V (Fig. 1 and Table 1). However, the unitary current of the double mutant Q98A/T338A was similar to that of the T338A single mutant. Interestingly, the unitary current of the K95Q/T338A double mutant exhibited a smaller single channel current amplitude $(0.09 \pm 0.01 \mathrm{pA})$ than that of either of its two single mutants, T338A (0.76 $\pm 0.01 \mathrm{pA})$ and K95Q (0.15 $\pm 0.01 \mathrm{pA})$, indicating a synergic effect of those two single mutants (Fig. 1 and Table 1). These results suggest that the residues K95 and T338 are functionally coupled and have a synergic effect on CFTR channel conductance.
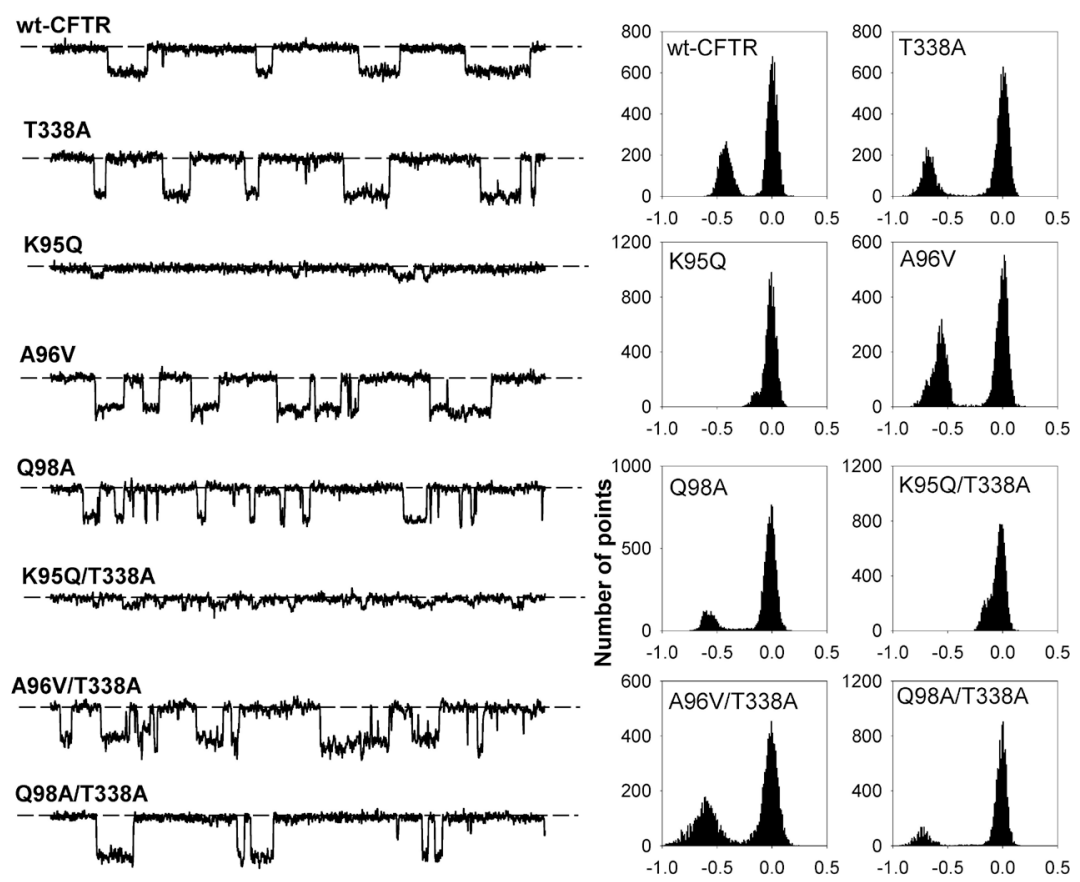

Fig. 1. Single channel current amplitudes of the wild type CFTR, single mutants in TM6 (T338A) and TM1 (K95Q, A96V and Q98A) and double mutants in TM1 and TM6 (K95Q/T338A, A96V/T338A, and Q98A/ T338A). Unitary currents were recorded in an inside-out configuration at a membrane potential of $-50 \mathrm{mV}$. For each trace, the short dashed line represents the current level when the CFTR channels are closed. The unitary current amplitudes are demonstrated in all point histograms (right panel) prepared from those current traces and as indicated. PKA (5 nM) and ATP

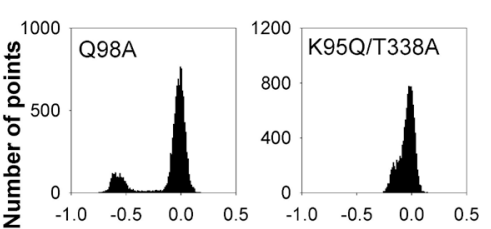
( $2 \mathrm{mM}$ ) were added to the bath solution to activate the CFTR channels.

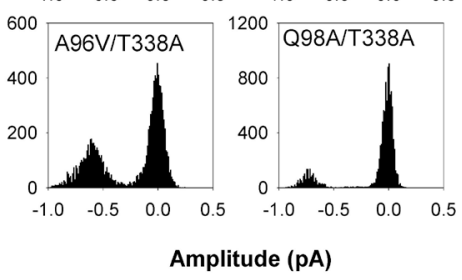

Table 1. Functional properties of CFTR wild type and mutant proteins.

\begin{tabular}{|c|c|c|c|c|c|}
\hline Mutants & $\begin{array}{l}\text { Unitary current } \\
\text { (pA) }\end{array}$ & $\begin{array}{c}\text { Rectification } \\
\text { ratio }\end{array}$ & $\begin{array}{c}\text { Relative } \\
\text { permeability } \\
\text { of } \mathrm{Br}^{-}\end{array}$ & $\begin{array}{c}\text { Relative } \\
\text { permeability } \\
\text { of } \mathrm{SCN}^{-}\end{array}$ & $\begin{array}{c}\text { Relative } \\
\text { permeability } \\
\text { of } \mathbf{I}^{-}\end{array}$ \\
\hline Wild type & $0.49 \pm 0.01$ & $1.05 \pm 0.02$ & $1.57 \pm 0.11$ & $2.08 \pm 0.04$ & $0.92 \pm 0.07$ \\
\hline T338A & $0.76 \pm 0.01^{\mathrm{a}}$ & $1.12 \pm 0.04$ & $2.00 \pm 0.07^{\mathrm{a}}$ & $7.50 \pm 0.56^{\mathrm{a}}$ & $3.17 \pm 0.37^{\mathrm{a}}$ \\
\hline$K 95 Q$ & $0.15 \pm 0.01^{\mathrm{a}}$ & $0.58 \pm 0.03^{a}$ & $1.44 \pm 0.23$ & $3.82 \pm 0.47^{\mathrm{a}}$ & $1.25 \pm 0.04^{\mathrm{a}}$ \\
\hline$A 96 \mathrm{~V}$ & $0.50 \pm 0.02$ & $1.56 \pm 0.02^{\mathrm{a}}$ & $1.54 \pm 0.04$ & $4.47 \pm 0.44^{\mathrm{a}}$ & $1.67 \pm 0.09^{\mathrm{a}}$ \\
\hline$Q 98 A$ & $0.51 \pm 0.01$ & $1.76 \pm 0.02^{\mathrm{a}}$ & $1.22 \pm 0.13^{\mathrm{a}}$ & $3.52 \pm 0.21^{\mathrm{a}}$ & $0.69 \pm 0.08^{a}$ \\
\hline T338A/K95Q & $0.09 \pm 0.01^{\mathrm{b}}$ & $0.23 \pm 0.02^{b}$ & $1.65 \pm 0.27$ & $5.09 \pm 0.11^{b}$ & $2.92 \pm 0.18$ \\
\hline T338A/A96V & $0.62 \pm 0.02^{b}$ & $1.47 \pm 0.05^{b}$ & $2.01 \pm 0.03$ & $7.01 \pm 0.18^{b}$ & $3.06 \pm 0.14$ \\
\hline T338A/Q98A & $0.75 \pm 0.01$ & $1.65 \pm 0.06^{b}$ & $1.81 \pm 0.09^{b}$ & $7.32 \pm 0.09$ & $3.23 \pm 0.46$ \\
\hline
\end{tabular}

Data are presented as means \pm SEM of 4-7 patches. The unitary currents were recorded at a membrane potential of $-50 \mathrm{mV}$. The value was obtained from the histograph of the unitary recording trace for each patch. The rectification ratio was quantified by the macroscopic slope conductance at $-50 \mathrm{mV}$ as a fraction of that at $+50 \mathrm{mV}$. The relative permeabilities $\left(P_{\mathrm{x}} / P_{\mathrm{Cl}}\right)$ of $\mathrm{Br}^{-}$, $\mathrm{SCN}^{-}$and $\mathrm{I}^{-}$in wild type CFTR and mutant proteins were calculated using Goldman-Hodgkin-Katz voltage equation: $P_{\mathrm{A}} / P_{\mathrm{B}}=\exp \left(\mathrm{E}_{\mathrm{Rev}} \mathrm{F} / \mathrm{RT}\right)$, where $\mathrm{E}_{\mathrm{Rev}}$ is the estimated current reversal potential; $F$ is the Faraday constant $\left(9.648 \times 10^{4} \mathrm{C} / \mathrm{mol}\right) ; R$ is the gas constant $(8.314 \mathrm{~J} / \mathrm{K} \cdot \mathrm{mol})$; and $\mathrm{T}$ is the absolute temperature in Kelvin (K). The superscript letter "a" after the values of single mutated CFTR channels indicates statistically significant (Student's twotailed $t$-test, $P<0.05)$ difference from wild type CFTR. The superscript letter " $\mathrm{b}$ " after the values of co-mutated CFTR channels indicates statistically significant (one-way ANOVA and Turkey HSD, $P<0.05$ ) difference from their relative single mutated channels. 

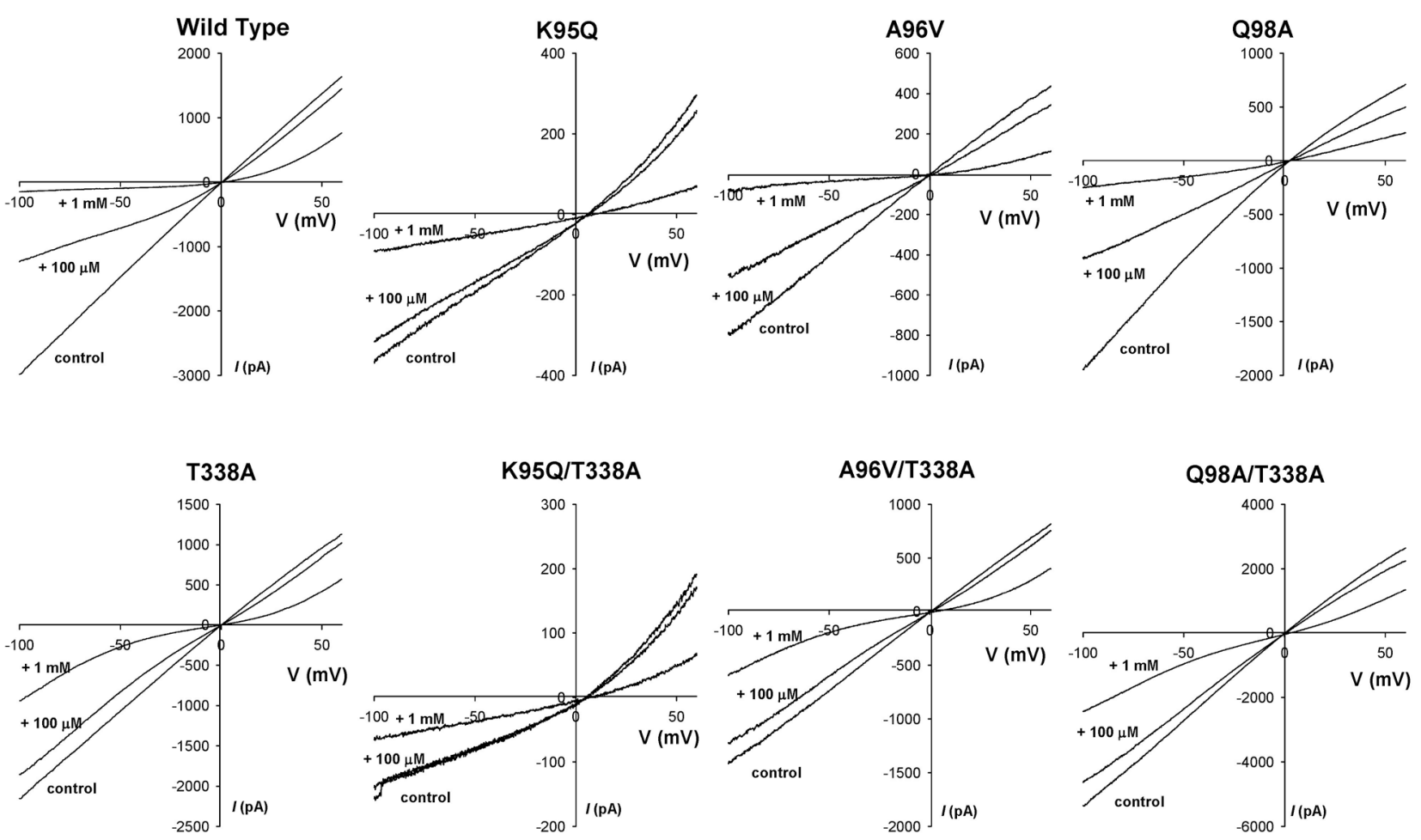

Fig. 2. $\mathrm{Au}(\mathrm{CN})_{2}^{-}$blockade of macroscopic CFTR currents. Examples of leak-subtracted macroscopic currents recorded in inside-out membrane patches following maximal activation with $50 \mathrm{nM}$ PKA, $2 \mathrm{mM}$ MgATP and $2 \mathrm{mM}$ sodium pyrophosphate under symmetrical $154 \mathrm{mM} \mathrm{Cl}^{-}$concentrations. In each case, currents were recorded before (Control) and after the addition of $100 \mu \mathrm{M} \mathrm{or} 1 \mathrm{mM} \mathrm{Au}(\mathrm{CN})_{2}{ }^{-}$to the intracellular solution.

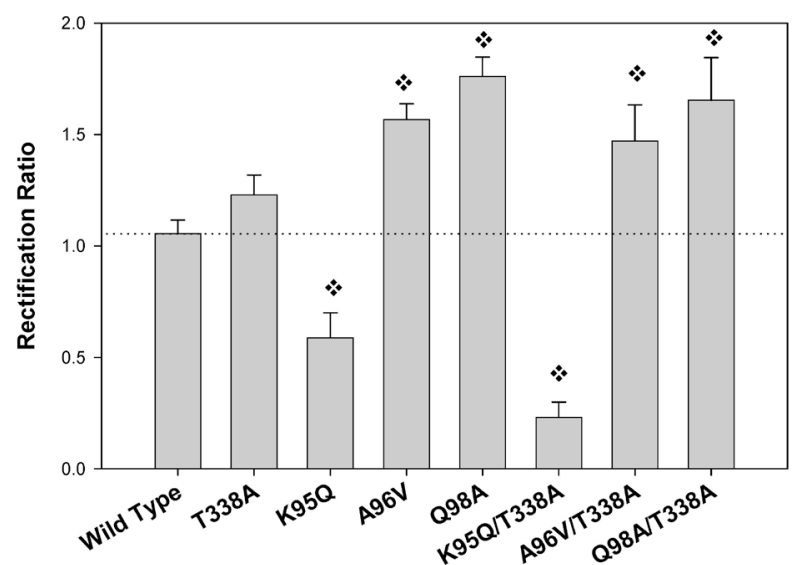

Fig. 3. Effect of mutations on rectification of the macroscopic $I-V$ relationship. Rectification was quantified as the rectification ratio as described in Materials and Methods, as the ratio of the slope conductance at $-50 \mathrm{mV}$ to that at $+50 \mathrm{mV}$. A ratio greater than one indicates inward rectification, and a ratio less than one indicates outward rectification of the $I-V$ curve. Asterisks indicate a significant difference from wild type $(P<0.05)$. Means \pm SEM were calculated from five to seven patches.

\section{Macroscopic current properties}

The macroscopic current properties were studied in inside-out patches following maximal channel activation in the presence of $2 \mathrm{mM}$ ATP, $50 \mathrm{nM}$ PKA and
$2 \mathrm{mM}$ pyrophosphate to keep the channels in the open configuration (Fig. 2). Examples of the leak-subtracted macroscopic CFTR $I-V$ relationships are shown in Figure 2 (control currents). Here, we used the rectification ratio (Gong and Linsdell 2003a, Ge et al. 2004) to compare the asymmetric permeation of $\mathrm{Cl}^{-}$ caused by mutations to the wild type CFTR channel (Fig. 2 and Fig. 3). In most cases (A96V, Q98A, A96V/T338A and Q98A/T338A), macroscopic $I-V$ relationships were inwardly rectifying (Table $1, P<0.05$ ) in the presence of symmetrical chloride concentrations, as shown in Figure 2 and Figure 3. Consistent with a previous report (Ge et al. 2004), K95Q showed strong outward rectification (Fig. 3). The K95Q/T338A double mutant demonstrated the characteristics of the single K95Q mutant. Significantly, the K95Q/T338A double mutant exhibited stronger outward rectification (a ratio of $0.23 \pm 0.02)$ than the K95Q single mutant $(0.58 \pm 0.03)$, even though the T338A single mutant showed inward rectification $(1.12 \pm 0.04)$.

To further investigate the CFTR mutants, we tested the blocking properties of $\mathrm{Au}(\mathrm{CN})_{2}{ }^{-}$, a high affinity probe for the anion binding sites in the CFTR pore (Gong et al. 2002a, Qian et al. 2014). In wild type CFTR 
chloride channels, the fractional unblocked current $\left(I / I_{0}\right)$ following addition of $100 \mu \mathrm{M}$ and $1 \mathrm{mM} \mathrm{Au}(\mathrm{CN})_{2}{ }^{-}$ increased in a voltage dependent manner when the voltage changed from $-100 \mathrm{mV}$ to $+60 \mathrm{mV}$ (Fig. 4). The fractional unblocked current of the T338A single mutant was also voltage-dependent, but it showed a U-shaped plot, with the highest blockade at a membrane potential close to zero (Fig. 4). $\mathrm{Au}(\mathrm{CN})_{2}{ }^{-}$was proposed to be capable of passing through the T338A single mutant channel under negative voltages, which suggests that the mutation reduced the binding affinity of $\mathrm{Au}(\mathrm{CN})_{2}{ }^{-}$to the channel pore. $\mathrm{Au}(\mathrm{CN})_{2}{ }^{-}$caused a voltage-dependent blockade of the wild type and mutant forms of CFTR at concentrations of $100 \mu \mathrm{M}$ and $1 \mathrm{mM}$, as demonstrated by the mean fractional I-V relationships (Fig. 2 and Fig. 4).
Decreased blockade and lower voltage dependence were observed in the K95Q, A96 V and Q98A single mutants and their corresponding double mutants, K95Q/T338A, A96V/T338A and Q98A/T338A compared to the T338A single mutant (Fig. 4). However, for the K95Q mutant, the fractional unblocked current $\left(I / I_{0}\right)$ in the presence of $\mathrm{Au}(\mathrm{CN})_{2}{ }^{-}$showed no significant voltage dependence for the voltage range tested. We also found that the K95Q/T338A double mutant exhibited very weak blockade of the membrane potential near $0 \mathrm{mV}$, weaker than those of the K95Q and T338A single mutants, while the Q98A/T338A double mutant exhibited weaker blockade at a more negative membrane potential (near $-100 \mathrm{mV}$ ) than its two single mutants Q98A and T338A (see the third row panel in Fig. 4).
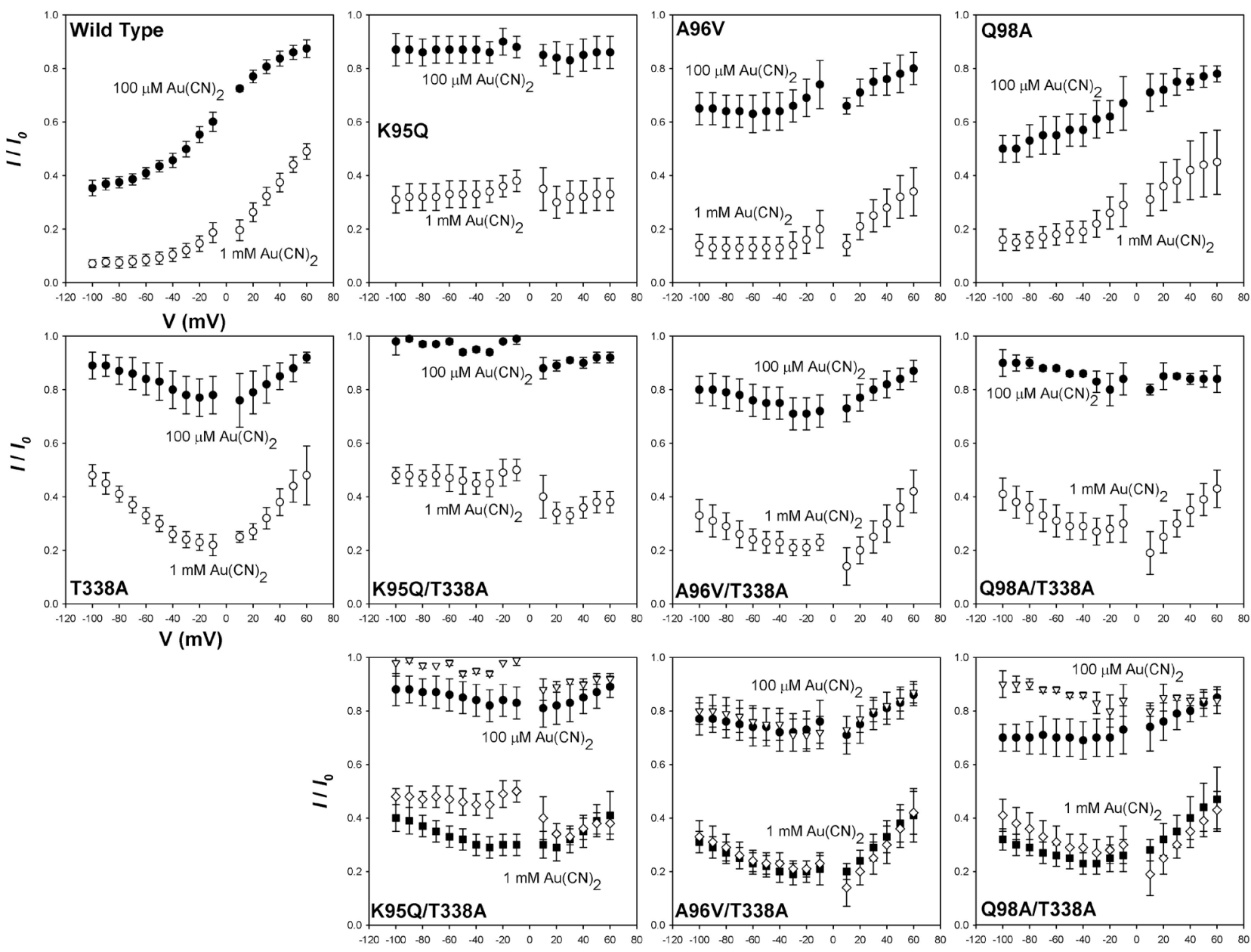

$\mathrm{V}(\mathrm{mV})$

Fig. 4. Effects of mutations on voltage-dependent blockade by $100 \mu \mathrm{M}$ and $1 \mathrm{mM}$ intracellular $\mathrm{Au}(\mathrm{CN})_{2}{ }^{-}$. Each panel in the first two rows shows the mean fractions of control current remaining ( $\left.\mathrm{I} / \mathrm{I}_{0}\right)$ following addition of $100 \mu \mathrm{M}$ (solid circles) or $1 \mathrm{mM}$ (hollow circles) $\mathrm{Au}(\mathrm{CN})_{2}^{-}$to the intracellular solution as a function of membrane potential. Each panel in the third row shows the superimposed comparisons of the average remaining fraction of two single mutants (K95Q and T338A, A96V and T338A, Q98A and T338A; hollow triangles and diamonds) with their corresponding double mutants (K95Q/T338A, A96V/T338A, and Q98A/T338A; solid circles and squares). Note that the double mutants (K95Q/T338A and Q98A/T338A) show synergic effects (weaker than that of the associated individual mutants) on $\mathrm{Au}(\mathrm{CN})_{2}{ }^{-}$blockade under negative membrane potentials. Means $\pm \mathrm{SEM}$ were calculated from five to seven patches. 

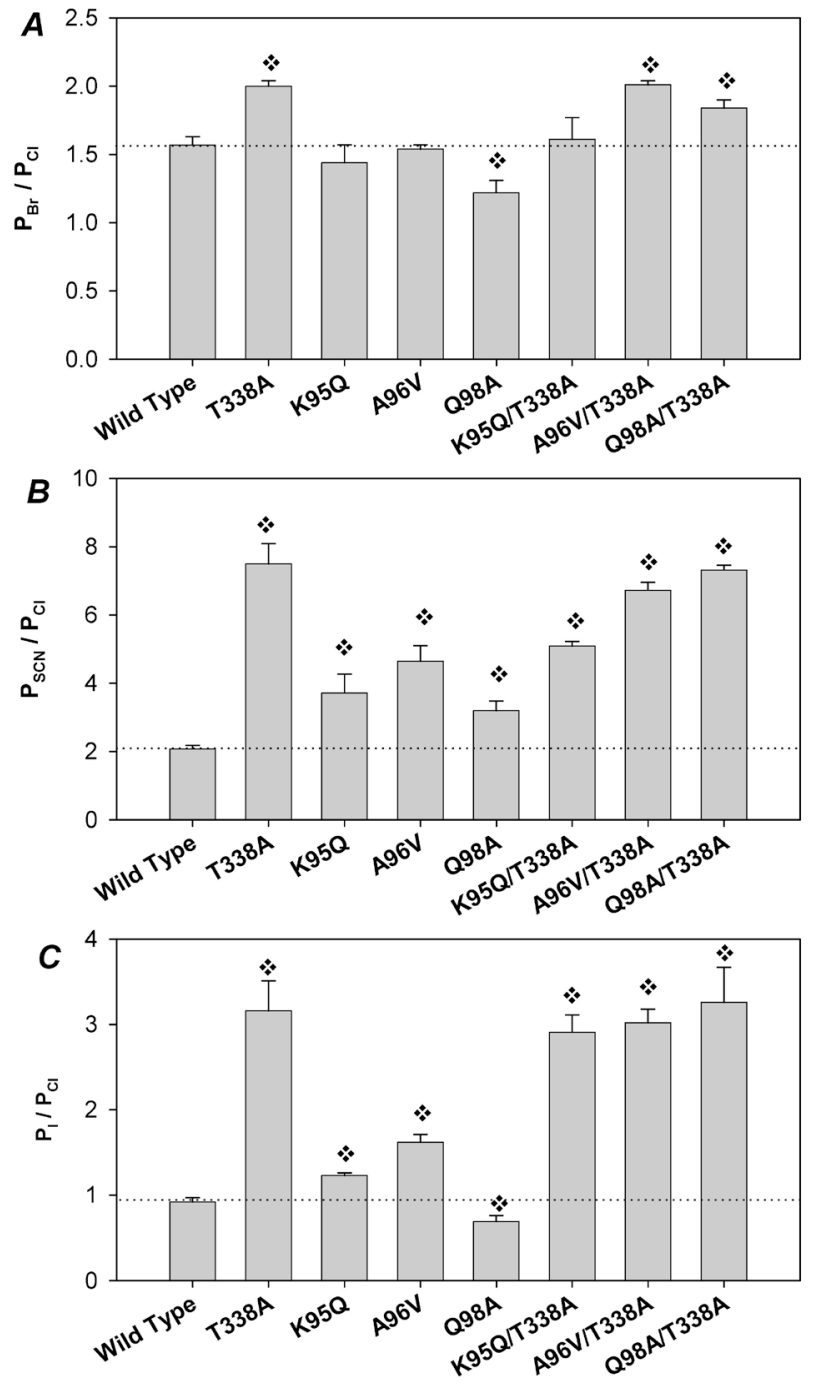

Fig. 5. Relative permeabilities $\left(P_{\mathrm{x}} / P_{\mathrm{Cl}}\right)$ of $\mathrm{Br}^{-}(\mathbf{A}), \mathrm{SCN}^{-}(\mathbf{B})$ and $\mathrm{I}^{-}(\mathbf{C})$ in wild type and CFTR mutants. $\mathrm{Br}^{-}$and $\mathrm{SCN}^{-}$were in the pipette solution (extracellular solution); however, $\mathrm{I}^{-}$was added to the bath solution (intracellular solution) to avoid electrode oxidation in the pipette, which can cause baseline drift. Asterisks indicate a significant difference from wild type $(P<0.05)$. Means \pm SEM were calculated from four to six patches.

\section{Anion selectivity}

CFTR is associated with a lyotropic anion selectivity pattern (Dawson et al. 1999) that has been shown to be disrupted by mutations in TM6 (Gong et al. 2002b). Mutagenesis studies of CFTR have also suggested that the selectivity of anion permeation is relatively resistant to the effects of mutagenesis unless the mutated residues are within the "selectivity filter" (Gong et al. 2002b). In the present study, we estimated the permeabilities of three anions $\mathrm{Br}^{-}, \mathrm{SCN}^{-}$, and $\mathrm{I}^{-}$based on the measurements of the macroscopic current reversal potential in inside-out excised patches. According to the reversal potentials under the bi-ionic condition, with anion $\mathrm{X}^{-}$-containing solution on one side of the membrane and $\mathrm{Cl}^{-}$-containing solution on the other side of the membrane, the relative permeability of $\mathrm{X}^{-}\left(P_{\mathrm{X}} / P_{\mathrm{Cl}}\right)$ was determined in wild type and mutated CFTR channels, and those permeability values are shown in Figure 5 and compared in Table 1. Based on that data, we found that all single mutants (K95Q, A96V, Q98A and T338A) played important roles in the relative permeability of the anions tested. However, the T338A mutant played a dominant role on CFTR anion selectivity in all three CFTR double mutants containing that mutation. Bromide permeability was increased in the T338A single mutant and double mutants containing T338A (A96V/T338A and Q98A/T338A), while it was reduced in the Q98A mutant (Fig. 5A). Thiocyanate permeability was increased at different levels in all mutants (Fig. 5B). The pattern of iodide permeability was similar to that of bromide permeability, but the mutants showed more sensitivity to iodide than to bromide (Fig. 5C).

\section{Discussion}

We constructed three CFTR double mutants, all with one mutation each in TM1 and TM6 (K95Q/T338A, A96V/T338A, and Q98A/T338A) to test our hypothesis that if the mutated residues are functionally coupled they may collaborate with each other and, thus, may influence the functional properties of CFTR channels. A prerequisite for employing a double mutant to examine the pore architecture of the CFTR channel is to identify at least one residue that clearly contributes to CFTR pore formation and if mutated causes a noticeable change in CFTR properties from those of wild type CFTR channels. We selected a background single mutation in TM6 because that TM region has a dominant role in CFTR channel pore formation, as indicated by the effects of site-directed mutations in TM6 on anion binding and permeation (Tabcharani et al. 1993, McDonough et al. 1994), current rectification (Gong and Linsdell 2003b, Ge et al. 2004), and blockade by CFTR channel blockers (Gong et al. 2002a,b, Linsdell 2014b). Among the identified pore-lining residues (R334, K335, F337, T338, S341, I344, V345, R347, and R352) in TM6, the polar, uncharged threonine residue (T338) stands out, as it has been implicated in the anion binding (Gong and Linsdell 2003b), single channel conductance (Linsdell et al. 1998) and anion selectivity (Linsdell et al. 1998, McCarty and Zhang 2001) properties of the pore, possibly by being 
located in the narrowest region of the pore (Linsdell et al. 1998, McCarty and Zhang 2001, Linsdell 2006). Therefore, we used T338A as the control CFTR channel variant and constructed all double mutations on this background. Our previous results regarding the stoichiometry of the CFTR channel (Qian et al. 2014) and those of other researchers (Marshall et al. 1994, Chen et al. 2002) suggest that the CFTR channel pore is formed by TM regions from a single CFTR molecule. The CFTR channel pore is likely formed by multimers of TM regions rather than by a single TM region, as is the case for potassium channels (Doyle et al. 1998); thus, we proposed that at least one other TM region in addition to TM6 must play a role in CFTR channel pore formation. Among the transmembrane regions, TM1 is the most likely to cooperate with TM6 in channel pore formation (Akabas et al. 1994, Ge et al. 2004, Linsdell 2006, Wang et al. 2011), and substitutions of several amino acid residues, such as G91 (Akabas et al. 1994, Mansoura et al. 1998), K95 (Anderson et al. 1991, Ge et al. 2004), A96 (Ge et al. 2004), Q98 (Akabas et al. 1994, Ge et al. 2004), and P99 (Sheppard et al. 1996, Ge et al. 2004), have been shown to affect the functional properties of the CFTR channel. Here, we chose three residues in TM1 for mutagenesis, K95, A96 and Q98, as they had previous been proposed to be pore-lining residues (Ge et al. 2004, Linsdell 2014a).

Previous mutagenesis studies have shown that the positively charged side chain of K95 may be involved in attracting $\mathrm{Cl}^{-}$ions and, thus, may help form the $\mathrm{Cl}^{-}$ion binding site inside the CFTR channel pore (Linsdell 2005, Rubaiy and Linsdell 2015). Therefore, we chose the most extensively studied mutants at these two sites, T338A and K95Q, to construct a CFTR double mutant protein with the threonine at position 338 being substituted with alanine and the lysine at position 95 being substituted with glutamine simultaneously. A synergic effect is expected if these two residues are functionally coupled, which had previously been shown for the T338A/T339A (Linsdell et al. 1998, Qian et al. 2014) and T338A/S341A (Qian et al. 2014) double mutants. To examine possible synergistic effects, we compared the functional effects of CFTR single mutations, such as K95Q, A96V and Q98A, with the effects of CFTR double mutations using a range of specific functional assays for single channel conductance (Fig. 1), anion binding (Figs 2, 3, 4), and anion selectivity (Fig. 5). The data of these investigations are analyzed and summarized in Table 1. Our findings provide evidence that T338 in TM6 has significant synergic effects with K95 in TM1, and we propose that these two residues may contribute to the formation of selectivity filter in the CFTR channel.

We found that the structural change caused by the T338A mutation amplified the outward rectification effect of the K95Q single mutation (Fig. 3). There are two possible explanations for this phenomenon: first, anion influx increased under a positive membrane potential; second, anion efflux decreased under a negative membrane potential. Because the single channel current of the K95Q/T338A double mutant was smaller than that of the K95Q single mutant at $-50 \mathrm{mV}$ (Table 1), it can be concluded that the T338A mutation further reduces the anion efflux caused by the K95Q mutation. Unlike the additive effects of A96V and T338A and Q98A and T338A, which could be considered to be due to functional overlapping, the effects of the T338A mutation on the $\mathrm{K} 95 \mathrm{Q}$ single mutant were quite different from its effects on the wild type CFTR channel. As expected, our findings indicated that the T338 site in TM6 has a stronger collaboration with K95 in TM1 than with A96 or Q98, possibly due to a direct interaction between these two residues within the same anion binding site through hydrogen bonds or polar contacts. However, it is unclear how the T338A mutation reduces anion efflux in the K95Q mutant while increasing the single channel conductance in the wild type CFTR $\mathrm{Cl}^{-}$channel. Our hypothesis is that the loss of the positively charged side chain in the K95Q mutant blocked the recruitment of anions at the cytoplasmic side of the channel pore (Linsdell 2005, Wang et al. 2011, Rubaiy and Linsdell 2015) subsequently reducing anion efflux. T338 is an important residue in the anion binding site, and the hydroxyl group of T338 may help K95 to recruit anions. In the T338A single mutant CFTR channel, the major anion recruiter residue, $\mathrm{K} 95$, remained non-mutated, and the size of the anion binding site was widened by the T338A substitution, resulting in increased channel conductance. However, when the major anion recruiter residue K95 is dysfunctional, T338 should play a more important role in anion recruitment than in determining the size of the pore. Therefore, the T338A mutation should be expected to subdue the unitary conductance under the $\mathrm{K} 95 \mathrm{Q}$ mutant background, if we assume that K95 and T338 form an anion binding site together near the cytoplasmic side of the CFTR $\mathrm{Cl}^{-}$channel pore.

A96V was used as a control mutant, as it was expected to have no effect on the functional properties of 
the CFTR channel. However, the additional side chain added by the substitution at that residue may have contributed to a structural change in the nearby anion binding site that could have consequently affected the anion selectivity of the CFTR channel (Fig. 5). We also found that the effect of the T338A single mutation on the unitary current was compromised by simultaneous mutation of A96V, but the unitary current of the Q98A/T338A double mutant was the same as that of the T338A single mutant. Because this phenomenon could be explained by the functional complement of the two collaborative residues, we propose that the additional side chain added at the site 96 in TM1 may partially compensate the removed side chain at the site 338 in TM6.

The unusual functional properties of the double mutant $\mathrm{K} 95 \mathrm{Q} / \mathrm{T} 338 \mathrm{~A}$ in terms of single channel conductance, anion permeability, macroscopic current rectification and the binding affinity of the CFTR channel blocker $\mathrm{Au}(\mathrm{CN})_{2}{ }^{-}$are due to the strong collaboration between K95 in TM1 and T338A in TM6. Therefore, we conclude that the side chains of residue K95 in TM1 and residue T338 in TM6 may form the selectivity filter. The functional proximity firmly establishes the electrostatic nature of the synergic effects of these two residues. Two of our experimental findings indicate that the selectivity filter is lined by amino acid side chains. First, substituting threonine at 338 with alanine resulted in a significant broadening of the channel diameter, indicating that the amino acid side chain at this point sticks out into the pore and determines its narrowest passage (Fatehi et al. 2007). Second, cysteine introduced at position K95 reacted rapidly with MTS reagents, indicating that cysteine's thiol group protrudes into the water-filled pore (Akabas et al. 1994, Wang et al. 2011). R334 and S341 residues in TM6 together with residues in TM1, most likely Q98, P99 and G91, may constitute other anion binding sites, one in the outer mouth and another on the intracellular side of the CFTR channel, as has been demonstrated in a cysteine-less CFTR model (Wang et al. 2011). Consistent with previous reports (El Hiani and Linsdell 2010, 2012, Wang et al. 2011, Qian et al. 2014), our results reinforce the notion that TM1 and TM6 play important roles in forming the CFTR channel pore and determining its functional properties.

\section{Conflict of Interest}

There is no conflict of interest.

\section{Acknowledgements}

We would like to thank Dr. Paul Linsdell for providing the pIRES2-EGFP-CFTR plasmid. This work was supported by grants from the Sciences Foundation of the Hubei Provincial Department of Education (Q20141301 and Q20151308) to Dr. F. Qian and Dr. L. Liu, Hubei Province's Outstanding Medical Academic Leader Program to Dr. C. Lu.

\section{References}

AKABAS MH, KAUFMANN C, COOK TA, ARCHDEACON P: Amino acid residues lining the chloride channel of the cystic fibrosis transmembrane conductance regulator. J Biol Chem 269: 14865-14868, 1994.

ANDERSON MP, GREGORY RJ, THOMPSON S, SOUZA DW, PAUL S, MULLIGAN RC, SMITH AE, WELSH MJ: Demonstration that CFTR is a chloride channel by alteration of its anion selectivity. Science 253: 202-205, 1991.

BAI Y, LI M, HWANG TC: Dual roles of the sixth transmembrane segment of the CFTR chloride channel in gating and permeation. J Gen Physiol 136: 293-309, 2010.

BAI Y, LI M, HWANG TC: Structural basis for the channel function of a degraded ABC transporter, CFTR (ABCC7). J Gen Physiol 138: 495-507, 2011.

CHEN JH, CHANG XB, ALEKSANDROV AA, RIORDAN JR: CFTR is a monomer: biochemical and functional evidence. J Membr Biol 188: 55-71, 2002.

CUI G, SONG B, TURKI H, MCCARTY N: Differential contribution of TM6 and TM12 to the pore of CFTR identified by three sulfonylurea-based blockers. Pflügers Arch 463: 405-418, 2012.

DAWSON DC, SMITH SS, MANSOURA MK: CFTR: mechanism of anion conduction. Physiol Rev 79: S47-S75, 1999.

DOYLE DA, MORAIS CABRAL J, PFUETZNER RA, KUO A, GULBIS JM, COHEN SL, CHAIT BT, MACKINNON R: The structure of the potassium channel: molecular basis of $\mathrm{K}+$ conduction and selectivity. Science 280: 69-77, 1998. 
DUTZLER R, CAMPBELL EB, CADENE M, CHAIT BT, MACKINNON R: X-ray structure of a ClC chloride channel at 3.0 A reveals the molecular basis of anion selectivity. Nature 415: 287-294, 2002.

DUTZLER R, CAMPBELL EB, MACKINNON R: Gating the selectivity filter in ClC chloride channels. Science 300: 108-112, 2003.

EL HIANI Y, LINSDELL P: Changes in accessibility of cytoplasmic substances to the pore associated with activation of the cystic fibrosis transmembrane conductance regulator chloride channel. J Biol Chem 285: 32126-32140, 2010.

EL HIANI Y, LINSDELL P: Tuning of CFTR chloride channel function by location of positive charges within the pore. Biophys J 103: 1719-1726, 2012.

FATEHI M, ST AUBIN CN, LINSDELL P: On the origin of asymmetric interactions between permeant anions and the cystic fibrosis transmembrane conductance regulator chloride channel pore. Biophys J 92: 1241-1253, 2007.

GADSBY DC, DOUSMANIS AG, NAIRN AC: ATP hydrolysis cycles and the gating of CFTR Cl- channels. Acta Physiol Scand Suppl 643: 247-256, 1998.

GAO X, BAI Y, HWANG T-C: Cysteine scanning of CFTR's first transmembrane segment reveals its plausible roles in gating and permeation. Biophys $J$ 104: 786-797, 2013.

GE N, MUISE CN, GONG X, LINSDELL P: Direct comparison of the functional roles played by different transmembrane regions in the cystic fibrosis transmembrane conductance regulator chloride channel pore. J Biol Chem 279: 55283-55289, 2004.

GONG X, LINSDELL P: Coupled movement of permeant and blocking ions in the CFTR chloride channel pore. J Physiol 549: 375-385, 2003a.

GONG X, LINSDELL P: Molecular determinants and role of an anion binding site in the external mouth of the CFTR chloride channel pore. J Physiol 549: 387-397, 2003b.

GONG X, BURBRIDGE SM, COWLEY EA, LINSDELL P: Molecular determinants of $\mathrm{Au}(\mathrm{CN})(2)(-)$ binding and permeability within the cystic fibrosis transmembrane conductance regulator $\mathrm{Cl}(-)$ channel pore. $J$ Physiol $\mathbf{5 4 0}$ : 39-47, 2002a.

GONG XD, LINSDELL P, CHEUNG KH, LEUNG GP, WONG PY: Indazole inhibition of cystic fibrosis transmembrane conductance regulator $\mathrm{Cl}(-)$ channels in rat epididymal epithelial cells. Biol Reprod 67: 1888$1896,2002 b$.

GUNDERSON KL, KOPITO RR: Effects of pyrophosphate and nucleotide analogs suggest a role for ATP hydrolysis in cystic fibrosis transmembrane regulator channel gating. J Biol Chem 269: 19349-19353, 1994.

LINSDELL P: Location of a common inhibitor binding site in the cytoplasmic vestibule of the cystic fibrosis transmembrane conductance regulator chloride channel pore. J Biol Chem 280: 8945-8950, 2005.

LINSDELL P: Mechanism of chloride permeation in the cystic fibrosis transmembrane conductance regulator chloride channel. Exp Physiol 91: 123-129, 2006.

LINSDELL P: Functional architecture of the CFTR chloride channel. Mol Membr Biol 31: 1-16, 2014a.

LINSDELL P: State-dependent blocker interactions with the CFTR chloride channel: implications for gating the pore. Pflugers Arch 466: 2243-2255, 2014b.

LINSDELL P, ZHENG SX, HANRAHAN JW: Non-pore lining amino acid side chains influence anion selectivity of the human CFTR Cl- channel expressed in mammalian cell lines. J Physiol 512: 1-16, 1998.

MACKINNON R: Potassium channels. FEBS Lett 555: 62-65, 2003.

MANSOURA MK, SMITH SS, CHOI AD, RICHARDS NW, STRONG TV, DRUMM ML, COLLINS FS, DAWSON DC: Cystic fibrosis transmembrane conductance regulator (CFTR) anion binding as a probe of the pore. Biophys J 74: 1320-1332, 1998.

MARSHALL J, FANG S, OSTEDGAARD LS, O'RIORDAN CR, FERRARA D, AMARA JE, HOPPE H IV, SCHEULE RK, WELSH MJ, SMITH AE, CHENG SH: Stoichiometry of recombinant cystic fibrosis transmembrane conductance regulator in epithelial cells and its functional reconstitution into cells in vitro. J Biol Chem 269: 2987-2995, 1994.

MCCARTY NA, ZHANG ZR: Identification of a region of strong discrimination in the pore of CFTR. Am J Physiol Lung Cell Mol Physiol 281: L852-L867, 2001. 
MCDONOUGH S, DAVIDSON N, LESTER HA, MCCARTY NA: Novel pore-lining residues in CFTR that govern permeation and open-channel block. Neuron 13: 623-634, 1994.

PILEWSKI JM, FRIZZELL RA: Role of CFTR in airway disease. Physiol Rev 79: S215-S255, 1999.

QIAN F, EL HIANI Y, LINSDELL P: Functional arrangement of the 12th transmembrane region in the CFTR chloride channel pore based on functional investigation of a cysteine-less CFTR variant. Pflugers Arch 462: 559-571, 2011.

QIAN F, LI T, YANG F, LIU L: Stoichiometry and novel gating mechanism within the cystic fibrosis transmembrane conductance regulator channel. Exp Physiol 99: 1611-1623, 2014.

RUBAIY HN, LINSDELL P: Location of a permeant anion binding site in the cystic fibrosis transmembrane conductance regulator chloride channel pore. J Physiol Sci 65: 233-241, 2015.

SHEPPARD DN, WELSH MJ: Structure and function of the CFTR chloride channel. Physiol Rev 79: S23-S45, 1999.

SHEPPARD DN, TRAVIS SM, ISHIHARA H, WELSH MJ: Contribution of proline residues in the membranespanning domains of cystic fibrosis transmembrane conductance regulator to chloride channel function. $J$ Biol Chem 271: 14995-15001, 1996.

TABCHARANI JA, ROMMENS JM, HOU YX, CHANG XB, TSUI LC, RIORDAN JR, HANRAHAN JW: Multi-ion pore behaviour in the CFTR chloride channel. Nature 366: 79-82, 1993.

VERGANI P, LOCKLESS SW, NAIRN AC, GADSBY DC: CFTR channel opening by ATP-driven tight dimerization of its nucleotide-binding domains. Nature 433: 876-880, 2005.

WANG W, EL HIANI Y, LINSDELL P: Alignment of transmembrane regions in the cystic fibrosis transmembrane conductance regulator chloride channel pore. J Gen Physiol 138: 165-178, 2011.

WANG W, EL HIANI Y, RUBAIY HN, LINSDELL P: Relative contribution of different transmembrane segments to the CFTR chloride channel pore. Pflugers Arch 466: 477-490, 2014. 